\title{
An E-HICH Structure for Enhanced Uplink in LCR TDD
}

\author{
Lijuan Wang \\ Dept. of Computer Science of Taiyuan University of \\ Technology \\ Taiyuan, China
}

\author{
Mingguang Ouyang \\ Huawei Technology Corporation \\ Beijing, China
}

\begin{abstract}
A reasonable E-HICH channel structure will consume the least system resource but possess the capacity of bearing the needed information while it could be shared by multiple UEs. In this paper, all the above factors are considered and an E-HICH structure for enhanced uplink in LCR TDD is designed.

Keywords-LCR TDD;E-HICH;Timeslot;Signature sequences;
\end{abstract} TPC/SS

\section{INTRODUCTION}

For HSUPA of LCR TDD, E-HICH is an important physical channel, which carries the HARQ acknowledgement indicators or TPC/SS command for uplink E-PUCH[1]. Therefore E-HICH channel structure is the key element in HSUPA of LCR TDD. The similar channel has been designed in WCDMA and HCR TDD.

In LCR TDD, the code resource and power resource is precious. A reasonable E-HICH channel structure will consume the least system resource but possess the capacity of bearing the needed information. If a similar channel structure for WCDMA or HCR TDD is explored in LCR TDD[2], it will kill much related resource especially the downlink codes.

Another problem with E-HICH channel is that E-HICH is shared by multiple UEs. The multiplexed means should be considered while the channel structure is designed.

The related working groups in $3 \mathrm{GPP}$ discuss the specification 25.827 and 30.302[3]. Some schemes are proposed to 3GPP for discussion such as CATT, TD-TECH.

In CATT proposal, E-HICH is a $\mathrm{SF}=16$ downlink physical channel and QPSK modulation shall be applied. Multiple users' ACK/NACK indicators are code-division-multiplexed on E-HICH[4]. Scheduled users' ACK/NACK indicators are carried on different E-HICH from non-scheduled users. E-HICHs for non-scheduled users carry not only ACK/NACK indicators but also TPC/SS commands.

In TD-TECH proposal, a multiplexed structure for E-HICH and E-AGCH is designed with the purpose to save power resource and to use beamforming technique.

Although these proposals have their respective advantages, their disadvantages are also obvious such as code consumption, UEs multiplexed problem[5]. Therefore, a more efficient E-HICH channel structure is needed.

\section{DESIGN OVERVIEW}

In LCR TDD, there are two different traffic types: scheduled traffic and non-scheduled traffic, which are carried on scheduled transmission and non-scheduled transmission respectively. E-PUCH[6] is used to bear these traffics. For scheduled traffic, the HARQ acknowledgement indicators ACK/NACK are transmitted on E-HICH. For non-scheduled traffic, the TPC/SS commands are required by UE besides the ACK/NACK. The scheduled traffic and non-scheduled traffic are transmitted in different TTI.

Since E-HICH is a shared physical channel, users are multiplexed on the same channel in a TTI. In order to save the code and power resource, a reasonable multiplexing means is needed for HSUPA in LCR TDD. The number of E-HICHs in a cell is configured by the system. Scheduled traffics' and non-scheduled traffics' acknowledgement indicators can be transmitted on the same E-HICH channel.

In this paper, The E-DCH Hybrid ARQ Indicator Channel $(\mathrm{E}-\mathrm{HICH})$ is a fixed rate $(\mathrm{SF}=16)$ downlink physical channel carrying the uplink E-DCH hybrid ARQ acknowledgement indicator and TPC/SS command[7]. QPSK modulation shall be applied and the following timeslot format is used:

Different users are multiplexed in an E-HICH channel through respective signature sequences, which are allocated by high layer signal. There are 40 signature sequences in total, each of which has the length of 40bits. The sequence bits can assume the value 1 or -1 . Different signature sequences can be allocated to the same user or to different users.

\section{DESIGN DETAILS}

\section{A. Scheduled traffic}

Scheduled traffic is transmitted on scheduled transmission. The HARQ acknowledgement indicators ACK/NACK are transmitted on E-HICH[8]. Table.2 is the mapping between ACK/NACK value and HARQ acknowledgement command.

The HARQ acknowledgement indicator $\alpha$ is spread by the assigned orthogonal signature sequence $C 1_{s s, 40, i, j}$, which is allocated by high layer. The output sequence $A_{s s, 40, i, j}$ equals to: 
$A_{s s, 40, i, j}=\alpha * C 1_{s s, 40, i, j}$

Where $j=0,1, \ldots, 39$

The output sequence $A_{s s, 40, i, j}$ has 40bits, which will experience the E-HICH spreading process with specific OVSF code. A 320chips will be achieved after the spreading process. They are assigned to data part 1.The filler chips are added to the left spare positions in the data part 1 . The data part 2 repeats the data content of data part 1 to achieve a higher performance. The following figure shows the sub-frame structure.

In a TTI for scheduled traffic, only one signature sequence is needed for a specific user.

\section{B. Non-scheduled traffic}

Non-scheduled traffic is transmitted on non-scheduled transmission. The HARQ acknowledgement indicators ACK/NACK are transmitted on E-HICH. The mapping relation is the same as that of scheduled traffic[9], shown in table3.

The HARQ acknowledgement indicator $\beta$ is spread by the assigned orthogonal signature sequence $C 1_{s s, 40, i, j}$, which is allocated by high layer. It is the same as the one for scheduled traffic. The output sequence $B_{s s, 40, i, j}$ equals to:

$B_{s s, 40, i, j}=\beta^{*} C 1_{s s, 40, i, j}$

Where $j=0,1, \ldots, 39$

The output sequence $B_{s s, 40, i, j}$ has $40 \mathrm{bits}$, which will experience the E-HICH spreading process with specific OVSF code. A 320chips will be achieved after the spreading process. They are assigned to data part 1.The filler chips are added to the left spare positions in the data part 1 . The data part 2 repeats the data content of data part 1 to achieve a higher performance. The following figure shows the sub-frame structure.

The TPC command $\tau$ is processed by the same means. It is spread by another signature sequence $C 2_{s s, 40, i, j}$, which is allocated by high layer or inferred from $C 1_{s s, 40, i, j}$, the output sequence $T_{s s, 40, i, j}$ equals to:
$T_{s s, 40, i, j}=\tau * C 2_{s s, 40, i, j} \quad$ Where $j=0,1, \ldots, 39$

After E-HICH spread, 320chips are generated, which are assigned to data part 1 of E-HICH sub-frame as the following figure. The left spare positions are set to filler bits.

The SS command $s$ is processed by the same means. It is spread by signature sequence $C 2_{s s, 40, i, j}$, which is the same as that of TPC command, the output sequence $S_{s s, 40, i, j}$ equals to:

$$
S_{s s, 40, i, j}=s * C 2_{s s, 40, i, j} \quad \text { Where } j=0,1, \ldots, 39
$$

After E-HICH spread, the generated 320chips are assigned to data part 2 of E-HICH sub-frame. The left spare positions are set to filler bits[10]. The following figure shows the structure.

In a TTI for non-scheduled traffic, two signature sequences are needed for a specific user. One is for $\mathrm{ACK} / \mathrm{NACK}$, the other is for TPC/SS command.

\section{RESULTS}

Based on this proposal, only one E-HICH is needed to transmit the ACK/NACK, TPC and SS, which not only save the code resource, but also save the power resource. From the following simulation result, a good performance can be achieved from our proposal.

\section{REFERENCES}

[1] 3GPP TS 25.211: "Physical channels and mapping of transport channels onto physical channels (FDD)".

[2] 3GPP TS 25.212: "Multiplexing and channel coding (FDD)".

[3] 3GPP TS 25.213: "Spreading and modulation (FDD)".

[4] 3GPP TS 25.215: "Physical layer - Measurements (FDD)".

[5] 3GPP TS 25.331: "RRC Protocol Specification".

[6] 3GPP TS 25.433: "UTRAN Iub Interface NBAP Signalling".

[7] 3GPP TS 25.101: "UE Radio transmission and Reception (FDD)".

[8] 3GPP TS 25.133: "Requirements for Support of Radio Resource Management (FDD)"

[9] 3GPP TS 25.321: " MAC protocol specification".

[10] 3GPP TS 25.306: "UE Radio Access Capabilities" 
TABLEI. E-HICH TIMESLOT FORMAT

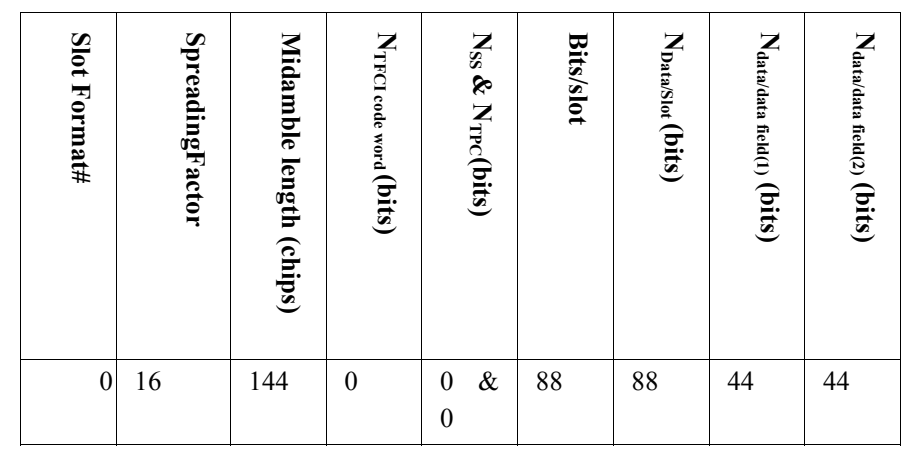

TABLEII. MAPPING BETWEEN ACK/NACK VALUE AND HARQ ACKNOWLEDGEMENT COMMAND

\begin{tabular}{|c|c|}
\hline $\begin{array}{c}\text { HARQ acknowledgement } \\
\text { command }\end{array}$ & value \\
\hline ACK & 1 \\
\hline NACK & 0 \\
\hline
\end{tabular}

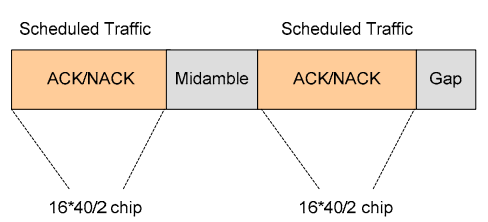

Figure1. ACK/NACK transmission for scheduled traffic

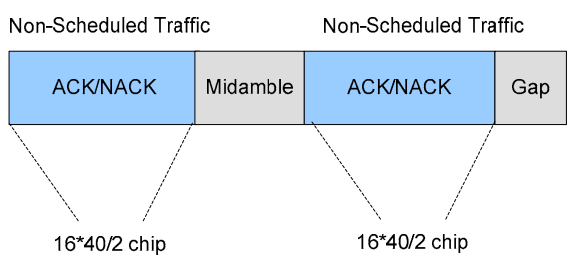

Figure2. ACK/NACK transmission for non-scheduled traffic

[11]

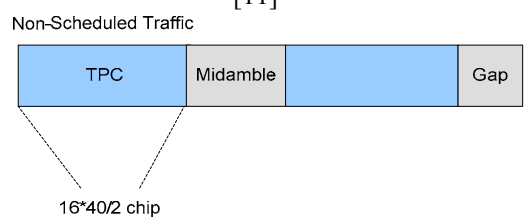

Figure3. TPC command for non-scheduled traffic 


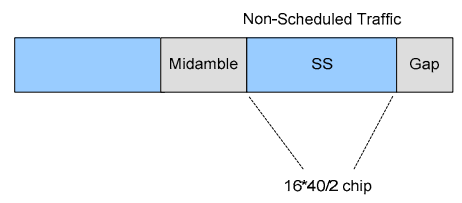

Figure4. SS command for non-scheduled traffic

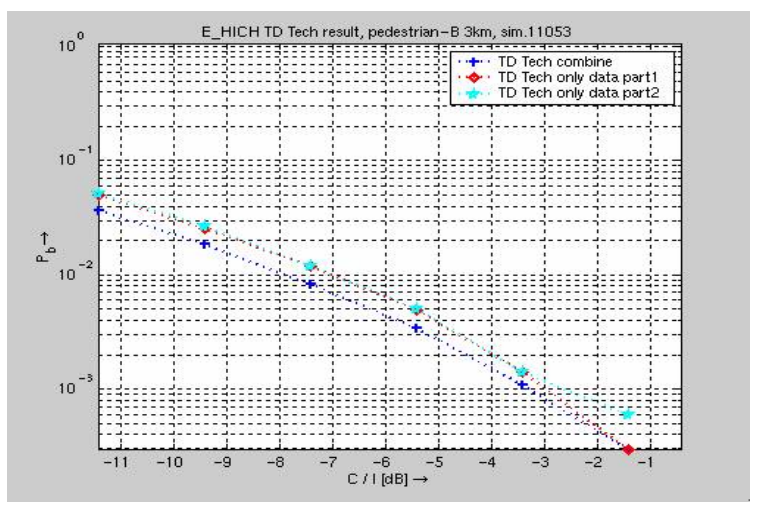

Figure5. Simulation result for ACK/NACK, TPC and SS 